\title{
SIMMETRIA PARITÀ-TEMPO IN OTTICA: VERSO UNA NUOVA INGEGNERIA DELLA LUCE
}

\author{
STEFANO LONGHI $(*)$ \\ Nota presentata dal m.e. Orazio Svelto \\ (Adunanza del 22 dicembre 2016)
}

\begin{abstract}
SuNTO. - L'introduzione in ottica dei concetti di simmetria parità-tempo, ben noti nelle teorie di meccanica quantistica e di teoria quantistica dei campi, sta rivoluzionando la nostra capacità di ideare e realizzare materiali ottici sintetici e dispositivi fotonici per il controllo della luce su micro- e nano-scala, con nuove funzionalità inimmaginabili con materiali ordinari e con importanti applicazioni nell' ambito delle tecnologie laser, della fotonica integrata e della sensoristica. In questa Nota verranno illustrati in maniera semplificata i principi teorici dell'ottica in materiali con simmetria parità-tempo e presentate le principali applicazioni alle tecnologie fotoniche.
\end{abstract}

$* * *$

ABSTRACT. - The introduction of the concept of parity-time symmetry in optics, inspired by recent theoretical developments in quantum mechanics and quantum-field theories, is revolutionizing our ability to design and manufacturing synthetic optical materials and photonic devices for molding the flow of light at the micro- and nano-scale, with novel functionalities impossible with ordinary materials and with important applications in the fields of laser technologies, integrated photonics and sensor technology. In this note I will illustrate the basic theoretical grounds of optical materials with paritytime symmetry and I will present the main recent applications to photonic technologies.

\section{INTRODUZIONE E MOTIVAZIONI}

Il concetto di simmetria è fondamentale in molti ambiti della scienza, ed ha attratto grande interesse fin dall'antichità da parte di

(*) Dipartimento di Fisica, Politecnico di Milano, Milano, Italia.

E-mail: stefano.longhi@polimi.it 
diversi pensatori, filosofi, artisti e scienziati [1]. Nella fisica moderna, i concetti di simmetria e di rottura spontanea di simmetria giocano un ruolo fondamentale e sono basilari per una comprensione moderna delle leggi fisiche. Basti pensare che le più grandi scoperte della fisica teorica del secolo XX, a partire dalla teoria della relatività ristretta fino alle teorie della superfluidità e della superconduttività nella fisica della materia ed alla teoria unificata delle interazioni elementari [2], possono essere interpretate in un certo senso come scoperte di invarianze, di simmetrie, e di rotture spontanee di simmetrie.

Alcune importanti simmetrie in Fisica sono legate a combinazioni di operatori quali la parità $P$, che possiamo identificare in maniera semplice con la simmetria spaziale di inversione, la coniugazione di carica $C$, che scambia ogni particella con la sua antiparticella (che ha carica opposta), e la trasformazione di inversione temporale $T$, che inverte la direzione del tempo (sostanzialmente come un film visto all'indietro). Attualmente si ritiene che le leggi fisiche debbano essere invarianti per simmetria TCP, e cioè che per ottenere invarianza delle leggi fisiche occorre scambiare, nelle immagini speculari degli eventi fisici, le particelle con le loro antiparticelle e guardare gli eventi nel loro fluire all'indietro.

Le simmetrie di parità $P$ e di tempo $T$ giocano un ruolo essenziale anche nell'ambito della meccanica quantistica non relativistica, dove la simmetria di coniugazione di carica non interviene. La teoria della meccanica quantistica ha ormai un secolo di vita e, in forza delle numerosissime verifiche sperimentali di tale teoria e della sua capacità di descrivere le proprietà della materia così come la osserviamo su scala microscopica (atomica), è comunemente accettata dalla scienza moderna. Uno degli assiomi centrali della meccanica quantistica è quello secondo cui ogni sistema fisico è descritto da un operatore autoaggiunto (o Hermitiano) $H$, il cosiddetto operatore Hamiltoniano del sistema, che ne compendia le sue proprietà intime e che consente di prevederne la sua evoluzione nel tempo. La proprietà menzionata, che in matematica implica l'uguaglianza dell'operatore Hamiltoniano con il suo aggiunto , resta da un punto di vista fisico piuttosto oscura, ma è considerata necessaria ed ineludibile per garantire alcuni elementari vincoli che il sistema deve soddisfare, come ad esempio il fatto che la particella non scompaia (conservazione della norma) e che le sue possibili energie siano quantità misurabili e quindi a valori reali.

Recentemente l'assioma che un sistema quantistico possa essere 
descritto da un operatore non Hermitiano è stata dibattuta in ambito teorico da alcuni autori, soprattutto in alcuni lavori di Carl Bender [3. 5]. In tali lavori, la condizione di Hermiticità viene sostituita dalla condizione (più debole) di simmetria parità-tempo $(P T)$ per l'operatore Hamiltoniano, e cioè che il commutatore si annulli. Poichè l'operatore di inversione temporale $T$ non è un operatore lineare, la proprietà non implica necessariamente che gli operatori Hamiltoniano e PT abbiano in comune uno stesso set di autofunzioni e autovalori. Si dice cha la simmetria PT non è rotta se le energie sono reali esattamente come per un operatore Hermitiano; solo quando c'è rottura della simmetria parità-tempo le energie diventano 'complesse'. La teoria avanzata da Bender è stata a lungo dibattuta e resta controversa, soprattutto perché in molti casi essa rappresenta una formulazione diversa ma equivalente alla formulazione ortodossa della meccanica quantistica mentre in altri casi pone difficoltà concettuali inerenti la violazione del principio 'nosignaling' di relatività (si veda, per esempio, [6]). Difatti, assumendo la simmetria PT (anziché l'Hermiticità dell'operatore Hamiltoniano) come fondamentale in una teoria quantistica, ci sarebbero radicali e nuove conseguenze, come la possibilità per un sistema quantistico di evolvere più rapidamente di quello previsto normalmente. In particolare, si potrebbe immaginare un esperimento in cui l'informazione viaggi più veloce della luce [6], il che è considerato impossibile dalla comunità scientifica in virtù delle limitazioni imposte dalla teoria della relatività ristretta di Einstein.

Come spesso accade in Fisica, fenomeni e sistemi molto diversi fra loro possono essere descritti da relazioni ed equazioni simili, e quindi possono mostrare dinamiche e fenomenologie simili. Analogie fra la meccanica quantistica (ondulatoria) e l'ottica sono ben note fin dai primordi delle teorie quantistiche della materia. Ad esempio, il principio di Fermat dell'ottica classica ed il principio della minima azione della meccanica classica esprimono sostanzialmente il medesimo principio per raggi luminosi e particelle classiche, e tale analogia fu usata per formulare una equazione ondulatoria per particelle, che ha dato origine alla cosiddetta meccanica ondulatoria (la forma primordiale della meccanica quantistica non relativistica). Un compendio recente di analogie fra ottica e meccanica quantistica, con applicazioni all'ottica ed alla fotonica integrata, può trovarsi nel lavoro [7]. La meccanica quantistica PT simmetrica trova una corrispondenza semplice ed elegante in ottica quando si considera la propagazione della luce in 
strutture dielettriche in cui l'indice di rifrazione del mezzo è immaginario, e più precisamente in cui si ha amplificazione e dissipazione di luce in maniera spazialmente bilanciata. Mentre teorie PT simmetriche in meccanica quantistica e teoria dei campi hanno mostrato serie criticità [6] e sembrano destinate a non avere sviluppi di particolare interesse [8], l'introduzione in ottica dei medesimi concetti di simmetria parità-tempo sta rivoluzionando, in soli pochi anni, la nostra capacità di ideare e realizzare materiali ottici sintetici e dispositivi fotonici. Materiali ottici con distribuzioni bilanciate di guadagno e perdite possono essere progettati in modo da controllare la propagazione della luce sulla microscala in maniera nuova rispetto ai metodi convenzionali, quali le guide e le fibre ottiche ordinarie, e sono di interesse in applicazioni alle tecnologie laser, alla fotonica integrata, alla sensoristica, alle tecniche di imaging. Nuovi sviluppi ed applicazioni dei concetti di simmetria parità-tempo all'ottica ed alle tecnologie laser si possono trovare in alcuni recenti lavori di letteratura, cui si rimanda il lettore per approfondimenti più specialistici [9-17]. In questa Nota verranno discusse brevemente ed in maniera semplificata alcune importanti applicazioni dei concetti di simmetria parità-tempo in ottica: la possibilità di realizzare un laser-antilaser, cioè un dispositivo capace simultaneamente di emettere luce coerente (come in un convenzionale oscillatore laser) e di assorbire completamente radiazione coerente incidente su di esso; la possibilità di costruire un metamateriale che mostra una invisibilità unidirezionale, che risulta cioè invisibile (come se esso non ci fosse) se osservato in una direzione dello spazio, ma non nella direzione opposta; infine la possibilità di generare luce laser strutturata, che trasporta momento angolare orbitale ('optical vortices'), in micro-laser ad anelli che operano ad un punto eccezionale

\section{SIMMETRIA PARITÀ-TEMPO IN OTTICA}

L'idea di applicare a strutture ottiche dielettriche proprietà di simmetria partà-tempo è piuttosto recente e risale ad alcuni lavori di pochi anni fa [18-21]. In ottica, una struttura con simmetria paritàtempo è sostanzialmente costituita da un materiale dielettrico in cui l'indice di rifrazione $n$ è complesso. Per ottenere un sistema ottico con simmetria $P T$, bisogna guardare all'indice di rifrazione da un punto di vista più generale e matematicamente più sottile di quanto non avvenga 
solitamente. Nel caso più generale, l'indice di rifrazione è infatti espresso da una quantità complessa, la cui parte reale è correlata al rallentamento della luce nel materiale e all'angolo con cui essa viene piegata, mentre la parte immaginaria determina l'amplificazione (guadagno) o l'assorbimento (perdita) di luce all'interno di un materiale. Un mezzo dielettrico con simmetria $P T$ ha sostanzialmente un profilo di indice di rifrazione reale che è una funzione pari per inversione, mentre la parte immaginaria dell'indice è una funzione dispari per inversione. In altri termini, il dielettrico può assorbire ed amplificare radiazione ottica in regioni diverse dello spazio ma in maniera bilanciata. In termini matematici, possiamo scrivere

$$
n(\boldsymbol{r})=n^{*}(-\boldsymbol{r})
$$

dove $\boldsymbol{r}$ è l'insieme delle coordinate spaziali. La propagazione della luce in tali strutture è descrivile mediante un operatore Hamiltoniano $H$ con simmetria $P T$, così come avviene nel problema quantistico. Ad esempio, nel caso più semplice in cui il mezzo dielettrico ha una indice di rifrazione $n(\boldsymbol{r})$ invariate in un a direzione dello spazio $z$ e che si discosta poco da un valore di riferimento (di substrato o bulk) $n_{s}$, la propagazione dell'onda luminosa nella direzione $z$ è descritta da una equazione parassiale simile all'equazione di Schoedinger per una particella quantistica non relativistica [7]

$\mathrm{i} \hbar \frac{\partial \psi}{\partial z}=H \psi$

dove $\hbar=\lambda /(2 \pi)$ è lunghezza d'onda normalizzata della luce, e l'operatore Hamiltoniano $H$ è legato alla distribuzione dell'indice di rifrazione complesso del mezzo secondo la relazione

$$
H=-\frac{\hbar^{2}}{2 n_{s}}\left(\frac{\partial^{2}}{\partial x^{2}}+\frac{\partial^{2}}{\partial y^{2}}\right)+n_{s}-n(r)
$$

Una distribuzione di indice di rifrazione che soddisfa la relazione (1) rende l'operatore H PT simmetrico. Quando la simmetria PT non è rotta, la luce si propaga nella struttura dielettrica senza che via sia, globalmente, assorbimento o amplificazione del segnale luminoso, sebbene fotoni vengano localmente creati e distrutti nel dielettrico. Quando la simmetria PT è rotta, vi è invece una amplificazione del segnale luminoso nella struttura, una situazione che generalmente viene detta instabilità ottica e che è alla base di fenomeni importanti quali l'emissione di luce laser per emissione stimolata dal rumore quantistico o di radiazione coerente in oscillatori ottici parametrici. 
La rottura di simmetria $P T$ è accompagnata dalla comparsa dei cosiddetti punti eccezionali o singolarità spettrali [22-25], che sono delle criticità matematiche dell'operatore $H$ nelle quali esso perde alcune proprietà importanti (come la completezza del suo spettro). Sono proprio tali criticità che modificano in maniera unica le proprietà di trasporto della luce e che rendono possibili alcuni fenomeni quali l'invisibilità unidirezionale di un mezzo materiale o la possibilità di realizzare un laser-antilaser, come discuterò nel seguito. Un'importante applicazione dei punti eccezionali nella tecnologia laser è quella, ad esempio, di selezionare in strutture integrate micrometriche l'oscillazione monomodale $[15,16]$. Due possibili schemi di strutture con simmetria parità-tempo per la selezione modale è mostrata nella Fig. $1[15,16]$. La prima consiste in una coppia di microanelli accoppiati per tunneling evanescente di luce, uno con guadagno e l'alto con perdite in modo da rispettare la condizione (1), mentre nella seconda struttura vi è un solo microanello con super-imposta una modulazione periodica delle perdite lungo l'anello. Nel primo caso, l'accoppiamento tra gli anelli con guadagno/perdite bilanciati induce un innalzamento del contrasto di estinzione dei modi longitudinali adiacenti dell'anello, rispetto al modo centrale che cade al centro della riga di guadagno del mezzo attivo, determinando così l'oscillazione su una singola risonanza longitudinale della cavità anche quando il laser emette molto al di sopra della sua soglia di oscillazione. Nel secondo caso la modulazione di perdite lungo l'arco dell'anello favorisce l'oscillazione di un singolo modo alla lunghezza d'onda che si sincronizza con il periodo della modulazione.

(a)

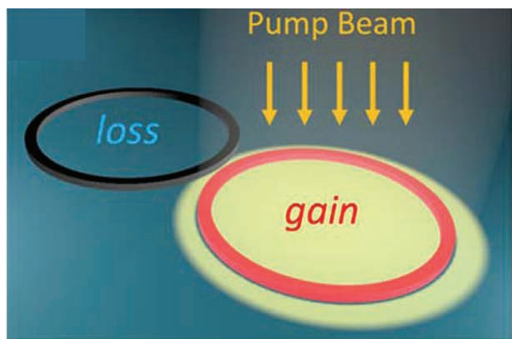

(b)

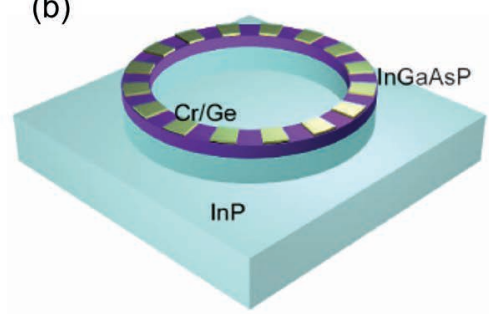

Fig. 1 - Schema di due strutture con simmetria parità-tempo in ottica integrata per la realizzazione di micro laser operanti in singolo modo.

(a) Microanelli accoppiati in modo evanescente, uno con perdite e l'altro con guadagno.

(b) Singolo micronaello con modulazione periodica delle perdite ottenuta mediante layer metallici di $\mathrm{Cr} / \mathrm{Ge}$ in semiconduttori InP/In GaAsP. 


\section{LASER ANTILASER CON SIMMETRIA PARITÀ-TEMPO}

Una delle conseguenze più interessanti della simmetria $P T$ applicata all'ottica è la possibilità di realizzare un dispositivo ottico attivo capace di emettere, come in un normale oscillatore laser, radiazione coerente che fuoriesce dal dispositivo ma in grado, simultaneamente, di assorbire in maniera perfetta una radiazione coerente che indice su di esso [10]. L'esistenza di un tale dispositivo sembrerebbe violare il senso comune secondo cui un mezzo materiale che amplifica luce e che quindi emette radiazione coerente quando posto in un cavità risonante (un laser) possa, simultaneamente, anche poter assorbire perfettamente luce coerente che incide su di esso (assorbitore coerente perfetto o antilaser). Tuttavia, l'esistenza di un tale dispositivo è una semplice e necessaria conseguenza della simmetria PT del mezzo ottico. Consideriamo, per semplicità, la propagazione della luce in una struttura ottica unidimensionale, ed indichiamo con $x$ la coordinata spaziale di propagazione e con $\varepsilon(x)=\varepsilon_{0} n^{2}(x)$ la constante dielettrica del mezzo spazialmente variabile in una regione di lunghezza $L$. La parte reale di $\varepsilon$ consente di localizzare la luce nella regione spaziale limitata di lunghezza $L$ (come ad esempio avviene nel caso di due specchi altamente riflettenti posti agli estremi della regione), mentre la parte immaginaria di $\varepsilon$ descrive i processi di assorbimento e/o emissione stimolata di fotoni che avviene nel mezzo materiale. Un'onda di luce di frequenza (generalmente complessa) $\omega$ è descritta da un campo elettrico $E=E(x, t)$ che soddisfa l'equazione di Helmholtz

$$
\frac{d^{2} E}{d x^{2}}+\frac{\omega^{2}}{c^{2}} \epsilon(x) E=0
$$

dove $c$ è la velocità della luce nel vuoto. L'equazione di Helmholtz, che è una conseguenza delle equazioni di Maxwell, è una equazione un po' più generale della equazione (2) introdotta nella precedente sezione, ma anche per essa possiamo introdurre degli operatori di inversione spaziale $(P)$ e temporale $(T)$. Supponiamo ora che il dispositivo sia una laser; allora la frequenza $\omega$ è reale e $E(x)$ descrive un'onda che fuoriesce dal mezzo, come indicato nel pannello di sinistra della Fig. 2. Se ora applichiamo alla equazione di Helmholtz l'operazione $T$ di inversione temporale, e cioè consideriamo il complesso coniugato della medesima equazione, le onde uscenti dal mezzo sono ora entranti e vengono totalmente assorbite da esso. La inversione temporale genera un nuovo mezzo materiale con costante dielettrica $\varepsilon^{*}(x)$, che rappresenta un assorbitore perfetto coerente (il flusso di energia rispetto al dispositivo laser è ora invertito): infatti la coniugazione trasforma un mezzo amplificatore in un mezzo assorbitore; si veda il pannello di destra della Fig. 2. 

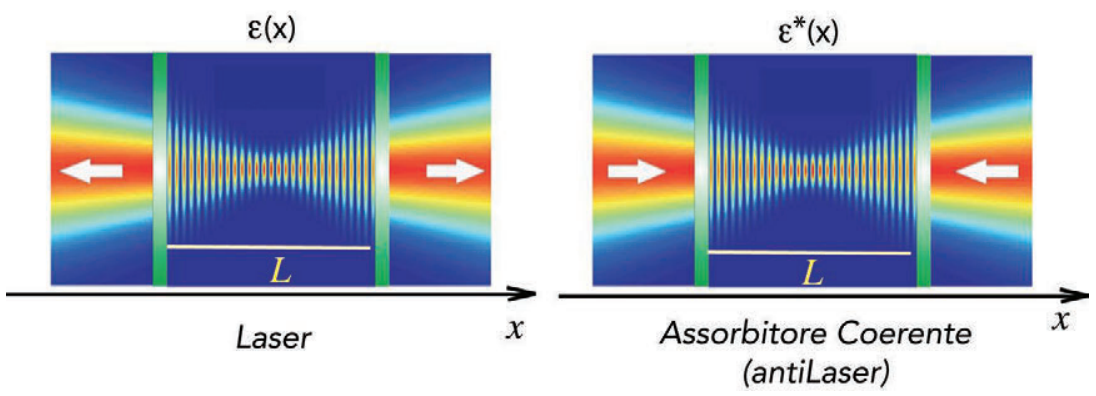

Fig. 2 - Principio di un dispositivo laser/antilaser basato sulle proprietà di simmetria parità-tempo. Il dispositivo a sinistra è un oscillatore laser che emette onde luminose uscenti dal mezzo (di lunghezza $L$ ). $\varepsilon(x)$ è la costante dielettrica del mezzo.

Se la costante dielettrica soddisfa la condizione di simmetria parità-tempo definita

dalla eq. (5), il medesimo mezzo può perfettamente assorbire onde coerenti incidenti su di esso, ottenute mediante inversione temporale e spaziale delle onde emesse nel caso del laser (come in un film avvolto all'indietro e visto specularmente).

Un'analisi più completa, basata su un approccio semiclassico dell'interazione luce-materia, è presentata nella referenza [26]. Se ora il mezzo ottico è invariante per simmetria $P T$, e cioè se

$\varepsilon(-x)=\varepsilon^{*}(x)$

è chiaro che il medesimo dispositivo che funziona come un laser deve anche funzionare come un assorbitore coerente perfetto. La condizione (5) sulla costante dielettrica implica che il mezzo dielettrico debba avere una distribuzione spazialmente bilanciata di perdite e guadagno (parte immaginaria della costante dielettrica). Possibili realizzazioni di sistemi laser/antilaser sono mostrate nella Fig. 3 e sono basate su strutture cosiddette a feedback distribuito, solitamente impiegate nella tecnologia dei laser a semiconduttore. Un struttura a feedback distribuito è sostanzialmente costituita da due mezzi semiconduttori trasparenti separati da una superficie che presenta una corrugazione periodica (modulazione) di periodo molto piccolo, dell'ordine della lunghezza d'onda di oscillazione. Tale corrugazione induce un accoppiamento di potenza tra le due onde contro-propaganti nel materiale (per un fenomeno noto come scattering o riflessione di Bragg) e consente così di intrappolare la luce al suo interno, agendo come uno specchio distribuito nello spazio. Per realizzare un dispositivo che possa simultaneamente operare come laser e come assorbitore coerente perfetto, metà del 
materiale assorbe luce, cioè distrugge fotoni con probabilità $\mathrm{P}_{1}$, mentre l'altra metà del materiale ne crea per emissione stimolata con una probabilità $\mathrm{P}_{2}$ esattamente uguale $\mathrm{a} \mathrm{P}_{1}$ [si veda il pannello (a) della Fig. 3]. Quando il valore di $\mathrm{P}_{1}=\mathrm{P}_{2}=\mathrm{P}$ è aumentato fino a raggiungere un valore critico, il sistema emette spontaneamente luce che esce nelle due direzioni opposte dello spazio (in prevalenza dalla parte dove il materiale amplifica luce), si è cioè al punto di rottura della simmetria $P T$ per la presenza di una singolarità spettrale o punto eccezionale. Ma se simultaneamente due onde luminose di opportuna ampiezza e fase relative incidono da parte opposte sulla struttura, i fotoni di queste onde vengono totalmente distrutti. Una struttura fotonica simile che può realizzare la medesima funzionalità è mostrata nel pannello (b) della Fig. 3, ed è costituita da un microdisco (o microanello) dielettrico nel quale vi è una corrugazione simultanea dell'indice di rifrazione e delle perdite, sfasate l'una dall'altra di un quarto di periodo. Va osservato che la possibilità di poter simultaneamente distruggere completamente luce di alcune onde incidenti, e creare fotoni per le rispettive onde speculari uscenti dal mezzo è certamente un fenomeno non intuitivo ma non è paradossale, ed è appunto una conseguenza della simmetria paritàtempo e spiegabile in ultima analisi mediante processi di interferenza delle onde nelle diverse regioni di perdite e guadagno nella struttura. $\mathrm{La}$ dimostrazione sperimentale del primo dispositivo laser-antilaser, teoricamente previsto nel lavoro [10], è stata recentemente riportata da un gruppo di ricerca americano usando tecnologia laser a semiconduttore e pubblicata sulla prestigiosa rivista Nature Photonics [28].

(a)

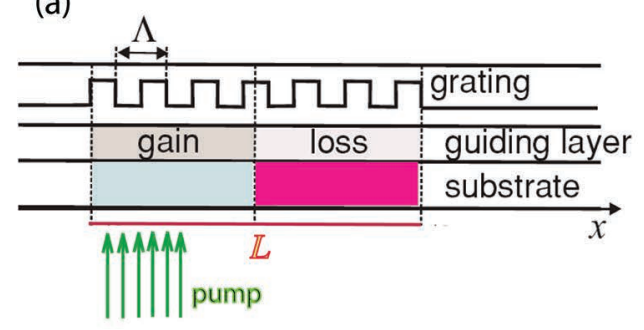

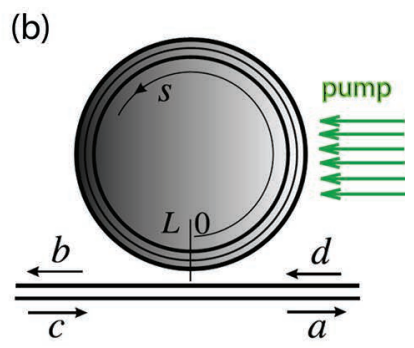

(b)

Fig. 3 - Esempi di strutture a feedback distribuito che realizzano un sistema laser/antilaser basato sul concetto di simmetria parità-tempo.

(a) Struttura a cavità lineare con reticolo di indice di rifrazione e con due regioni bilanciate di guadagno (zona verde) e perdite (zona rossa).

(b) Struttura ad anello (microanello accoppiato ad una guida d'onda di bus). L'anello presenta questa volta un reticolo di indice e di perdite. 


\section{INVISIBILITÀ UNIDIREZIONALE IN METAMATERIALI CON SIMMETRIA PARITÀ-TEMPO}

Un'altra interessante applicazione, ispirata dai concetti di paritàtempo, è la possibilità di realizzare metamateriali 'attivi', cioè che richiedono inversione di popolazione, che appaiono invisibili in funzione della direzione di provenienza della luce [29-31]. Una struttura che può diventare totalmente invisibile quando la luce la attraversa da un lato, mentre può rimanere visibile quando viene illuminata dall'altro, si basa sul fenomeno fisico della rottura di simmetria nel fenomeno dello scattering di Bragg che avviene quando la luce attraversa un mezzo periodico, come un cristallo. Nei cristalli naturali lo scattering di Bragg avviene in egual misura per la luce che si propaga in direzioni opposte. Ma se il cristallo è sintetizzato artificialmente in modo da descrivere un cristallo 'complesso' con simmetria parità-tempo del suo indice di rifrazione, al punto della rottura della simmetria PT lo scattering di Bragg è completamente assente quando la luce incide in una direzione del cristallo, ma non in quella opposta [21,24]; si veda la Fig. 4.

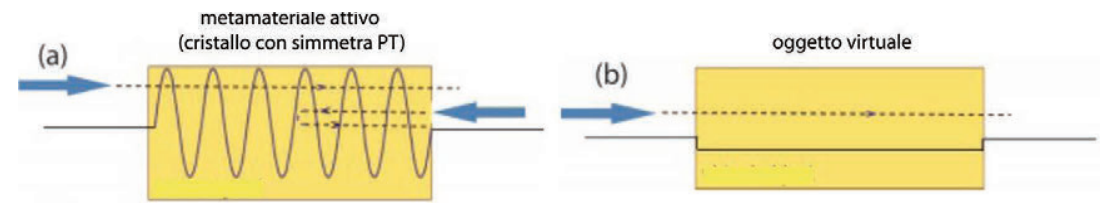

Fig. 4 - Principio dell'invisibilità unidirezionale in un metamateriale attivo con simmetria parità-tempo. Il metamateriale, mostrato schematicamente nel pannello (a) della figura, è costituito da un cristallo sintetico (meta-cristallo) che mostra una modulazione periodica dell'indice di rifrazione complesso. Quando le parti reale ed immaginarie dell'indice di rifrazione hanno la stessa ampiezze e sono sfasate di un quarto di periodo,

il meta-cristallo è al punto della rottura della simmetria PT e risulta invisibile per un'onda che si propaga in una direzione (da sinistra a destra nella figura), ma non in quella opposta. Per tale onda il cristallo appare invisibile, e precisamente ad un osservatore appare come un mezzo omogeneo che non riflette la luce [pannello (b) della figura].

Tale fenomeno è stato sperimentalmente dimostrato recentemente da due gruppi sperimentali usando come sistemi attivi guide dielettriche e fibre ottiche attive $[12,13]$; si veda la Fig. 5. Metamateriali capaci di rendere invisibili degli oggetti o, all'opposto, di ottenere una risoluzione delle immagini molto superiore al normale, sono già stati concepiti e dimostrati in ottica, ma nelle soluzioni precedentemente 
adottate l'alterazione attenta e specifica della distribuzione spaziale dell'indice di rifrazione all'interno del metamateriale era di tipo passivo e solitamente richiedeva strutture anisotrope e magnetiche [32].
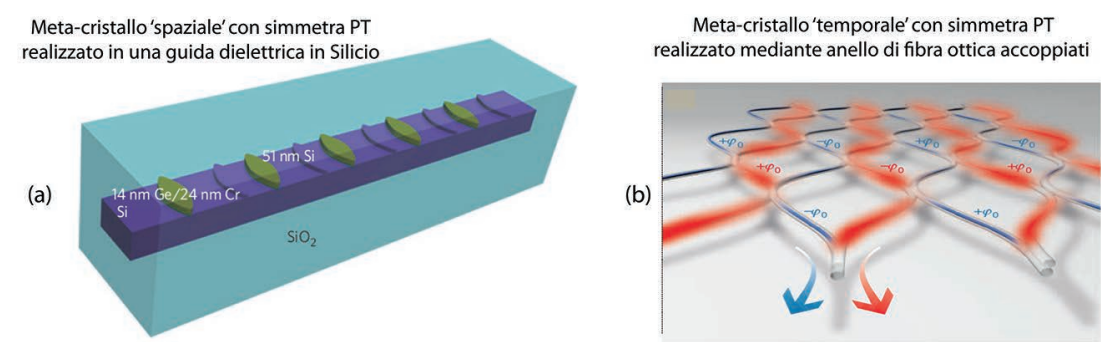

Fig. 5 - (a) Implementazione di un meta-cristallo con simmetria PT basato sulla propagazione spaziale di luce in una guida dielettrica di Silicio in SOI con reticolo di indice e di perdite sfasati di un quarto di periodo. (b) Implementazione di un meta-cristallo con simmetria PT basato sulla propagazione di impulsi ottici luminosi in anelli accoppiati di fibra ottica. Gli anelli sono disposti in una geometria simmetrica con alternanza di amplificazione e di perdite in anelli contigui.

Nelle strutture ottiche a simmetria parità-tempo, invece, il metamateriale è di tipo 'attivo', cioè richiede che l'indice di rifrazione sia complesso e vi sia una distribuzione bilanciata di perdite e guadagno. Le proprietà di invisibilità dei matemateriali attivi possono essere superiori di quelli dei soli metamateriali passivi [33], e questo apre quindi possibilità nuove di applicazioni all'imaging ottico. Inoltre, in tali mezzi è possibile introdurre forme parziali di invisibilità, ad esempio è possibile sintetizzare materiali sintetici che mostrano una invisibilità unidirezionale per metà dei colori della luce (ad esempio per lunghezze d'onda lunghe fino al rosso), ma non per l'altra metà (dal rosso alle lunghezze d'onda più corte) [34]. Una applicazione interessante della invisibilità unidirezionale, basata alla rottura di simmetria $P T$ ad un punto eccezionale, è quella della generazione di luce strutturata, che trasporta momento angolare orbitale, direttamente da un microlaser ad anello [35]. Tale tipo di luce, che trova importanti applicazioni ad esempio nella codifica e trasmissione di informazione ad altissimo bit rate, è costituita da fasci di luce vorticosi (vedi la Fig. 6), in cui cioè la parte centrale del fascio di luce è buio (zero di campo) mentre la fase del campo varia di un multiplo intero di $2 \pi$ (carica topologica del vortice) quando si percorre un cammino chiuso attorno al vortice. Luce vorticosa generata su scala micrometrica direttamente da un microla- 
ser ad anello richiede di poter forzare l'oscillazione laser nell'anello in una sola direzione (oraria o antioraria), per evitare che la vorticità del fascio emesso dall'anello vari stocasticamente seguendo le fluttuazioni aleatorie della oscillazione oraria-antioraria del laser innescata da processi non-lineari e di competizione modale nel microanello. Tale unidirezionalità richiede solitamente l'utilizzo di un isolatore ottico (diodo ottico) basato su effetti magneto-ottici, che sono tuttavia impraticabili su scale spaziali piccole (dell'ordine di qualche micrometro). L'uso di un reticolo combinato di indice e di perdite lungo il microanello, ingegnerizzato in modo da operare al punto della rottura della simmetria $P T$, consente di stabilizzare l'oscillazione unidirezionale del laser, senza l'utilizzo di un isolatore ottico di tipo magnetico (rotatorie di Faraday).

(a)

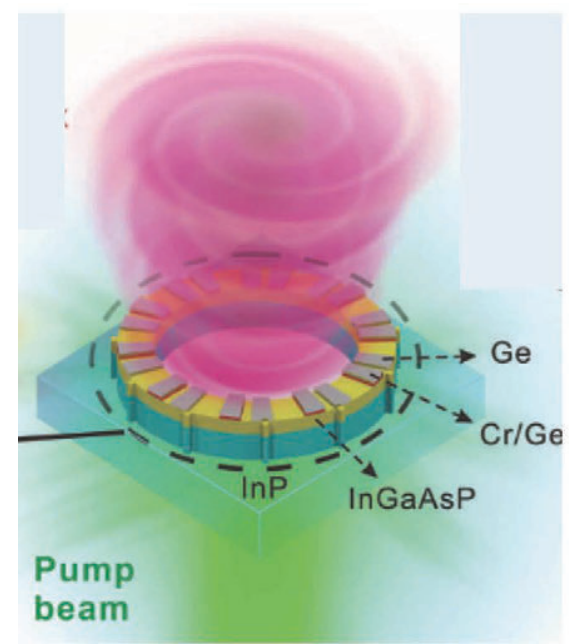

(b)
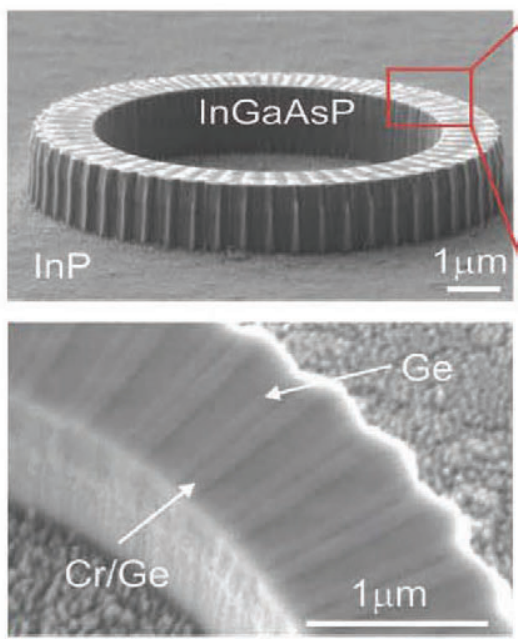

Fig. 6 - (a) Schema della generazione di luce strutturata (luce vorticosa che trasporta momento angolare orbitale) da un laser a microanello in cui l'oscillazione unidirezionale (oraria o antioraria) è ottenuta mediante l'uso di un cristallo PT simmetrico al punto di rottura della simmetria (punto eccezionale). (b) Microalaser in tecnologia semiconduttore

InP/In GaAsP che ba recentemente consentito la dimostrazione sperimentale della generazione di luce strutturata direttamente da un microanello del diametro di soli $9 \mu \mathrm{m}$.

Come osservazione conclusiva, si deve menzionare che il problema dell'invisibilità in mezzi attivi, qui illustrato con riferimento ai concetti di simmetria parità-tempo, può essere anche esteso a famiglie di 
sistemi ottici più generali che non godono di tale simmetria. Per esempio, utilizzando i concetti di supersimmetria noti nella meccanica quantistica, è possibile sintetizzare dei difetti invisibili in un cristallo, difetti che cioè non alterano le proprietà di propagazione dell'onda nel cristallo [36]. Questo risultato è piuttosto interessante e desueto, perché difetti reticolari solitamente producono scattering dell'onda. Recentemente, sono state anche scoperte ed investigate alcune proprietà interessanti, fra cui la possibilità di ottenere invisibilità unidirezionale o bidirezionale, in mezzi ottici nei quali le distribuzioni spaziali delle parti reali ed immaginarie dell'indice di rifrazione soddisfano certe relazioni speciali, le cosiddette relazioni di Kramers-Kronig [3740] note anche in matematica come trasformazioni di Hilbert. Queste relazioni, che sono ben note in ottica ed esprimono il principio di causalità nella risposata di ogni mezzo materiale esistente in natura, garantiscono l'assenza di onde riflesse quando il mezzo è illuminato da un lato, indipendentemente dall'angolo di incidenza della luce, ma solitamente non dal lato opposto.

\section{CONCLUSIONI}

In questa breve Nota si è discusso in maniera introduttiva e semplificata dei principi teorici e delle applicazioni dei concetti di simmetria parità-tempo in ottica, con particolare riguardo alla sintesi di materiali artificiali (metamateriali) che promettono interessanti sviluppi ed applicazioni nelle tecnologie laser, nella fotonica integrata, nel sensing e nelle tecniche di imaging ottico. L'ottica dei sistemi con simmetria parità-tempo è un settore di ricerca piuttosto recente ma molto promettente, che ha tratto molte idee e beneficiato di metodi di analisi sviluppati in meccanica quantistica. Oltre alla simmetria parità-tempo, altre proprietà di simmetria della meccanica quantistica e della teoria dei campi possono trovare interessanti applicazioni all'ottica. Menzioniamo brevemente, a titolo d'esempio, la simmetria di inversione temporale $T$, che trova applicazioni nella realizzazione di assorbitori coerenti anche policromatici [41], le proprietà di supersimmetria che possono essere ingegnosamente utilizzate per progettare circuiti fotonici integrati [42-44], ed altre proprietà di simmetria (come quella di chiralità) che garantiscono ad esempio l'esistenza di modi di propagazione di edge della luce insensibili al disordine della struttura 
a causa di una protezione di natura topologica (si tratta cioè di strutture fotoniche con proprietà simili ai cosiddetti isolanti topologici studiati nella Fisica dello stato solido). Con le teorie di supersimmetria è possibile costruire, per esempio, intersezioni trasparenti di guide dielettriche, cioè incroci di guide le quali non interferiscono le une con le altre ed i fotoni si propagano indipendentemente in esse (si veda la Fig. 7). Visto l'elevato interesse che tali tematiche hanno suscitato nella comunità scientifica internazionale, è prevedibile nei prossimi anni un rapido sviluppo e una maturazione tecnologica di queste ricerche che consentiranno di progettare e realizzare circuiti fotonici innovativi e materiali sintetici di nuova generazione con importanti applicazioni alla fotonica, alle tecnologie ottiche, alla sensoristica ed alle tecnologie dei laser.

(a)

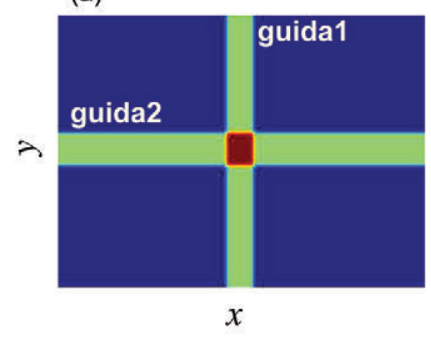

(b)

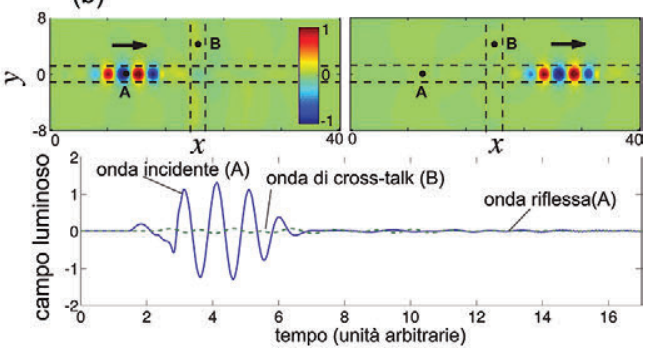

Fig. 7 - Intersezione trasparente di due guide ottiche basato sull'uso della supersimmetria della meccanica quantistica. (a) Schema di due guide di luce che si intersecano.

L'intersezione provoca generalmente un disturbo per la luce che si propaga nelle due guide. Tuttavia, se l'indice di rifrazione delle guide soddisfa a certe proprietà di supersimmetria, le onde luminose in ciascuna delle due guide attraversano l'intersezione senza subire riflessioni o senza disturbare il canale dell'altra guida. In sostanza l'intersezione appare trasparente. La trasparenza indotta da supersimmetria è mostrata nel pannello (b), in cui sono indicati l'andamento nel tempo di un segnale luminoso misurato nei punti A e B delle due guide. La trasparenza dell' intersezione si evince dal fatto che in B non si osserva luce (assenza del cosiddetto segnale di cross-talk) ed in A il segnale ritardato nel tempo (eco) corrispondente a riflessione di luce alla discontinuità praticamente assente.

\section{RINGRAZIAMENTI}

L'autore ringrazia la Pia Fondazione Edoardo Kramer e l'Istituto Lombardo Accademia di Science e Lettere per l'assegnazione del premio Edoardo Kramer 2015. 


\section{BIBLIOGRAFIA}

[1] E. Castellani, "Simmetria e Natura" (Laterza, Bari-Roma, 2001).

[2] "Simmetria e realtà" in: Le Scienze, Quaderni n. 118. A cura di E. Castellani, Febbraio 2001.

[3] C.M. Bender, S. Boettcher, "Real Spectra in Non-Hermitian Hamiltonians Having PT Symmetry”, Physical Review Letters (1998), vol. 80 pp. 5243-5246 DOI: 10.1103/PhysRevLett.80.5243.

[4] C.M. Bender, D.C. Brody, and H.F. Jones, "Complex Extension of Quantum Mechanics”, Physical Review Letters (2002), vol. 89, pp. 270401 - DOI: 10.1103/PhysRevLett.89.270401.

[5] C. Bender, "Making Sense of Non-Hermitian Hamiltonians", Reports Progress Physics (2007), vol. 70, pp. 947-1018 - DOI:10.1088/0034-4885/70/6/R03.

[6] Y.-C. Lee, M.-H. Hsieh, S.T. Flammia, and R.-K. Lee, "Local PT symmetry Violates the No- Signaling Principle”, Physical Review Letters (2014), vol. 112, pp. 130404 - DOI: 10.1103/PhysRevLett.112.130404.

[7] S. Longhi, "Quantum-optical analogies using photonic structures", Laser \& Photonics Review (2009), vol. 3, pp. 243-261 - DOI: 10.1002/lpor.200810055.

[8] M. Schirber, "Synopsis: Reflecting on an Alternative Quantum Theory", Physics (https://physics.aps.org/synopsis-for/10.1103/PhysRevLett.112.130404).

[9] C.E. Ruter, K.G. Makris, R. El-Ganainy, D.N. Christodoulides, and M. Segev, "Observation of parity-time symmetry in optics", Nature Physics (2010), vol.6, pp. 192-195 - DOI: 10.1038/nphys1515.

[10] S. Longhi, "PT -symmetric laser-absorber", Physical Review A (2010), vol. 82, pp. 031801- DOI: 10.1103/PhysRevA.82.031801.

[11] L. Feng, M. Ayache, J. Huang, Y.L. Xu, M.H. Lu, Y.F. Chen, Y. Fainman, and A. Scherer, "Nonreciprocal light propagation in a silicon photonic circuit," Science (2011), vol. 333, pp.729-733 - DOI: 10.1126/science.1206038.

[12] L. Feng, Y.-L. Xu, W. S. Fegadolli, M.-H. Lu, J.E.B. Oliveira, V. R. Almeida, Y.F. Chen, and A. Scherer, "Experimental demonstration of a unidirectional reflectionless parity-time metamaterial at optical frequencies", Nature Materials (2012), vol.12, 108-113 - DOI: 10.1038/nmat3495.

[13] A. Regensburger, C. Bersch, M.-A. Miri, G. Onishchukov, D.N. Christodoulides, and U. Peschel, "Parity-time synthetic optical lattices", Nature (2012), vol. 488, pp.167-171 - DOI: 10.1038/nature11298.

[14] L. Feng, Z.J. Wong, R.M. Ma, Y. Wang, and X. Zhang, "Single-mode laser by parity-time symmetry breaking”, Science (2014) vol. 346, pp. 972-975 - DOI: 10.1126/science. 1258479 .

[15] H. Hodaei, M.A. Miri, M. Heinrich, D.N. Christodoulides, and M. Khajavikhan, "Parity-time symmetric microring lasers", Science (2014), vol. 346, 975-978 DOI: $10.1126 /$ science. 1258480.

[16] R. Fleury, D. Sounas, and A. Alù, "Negative Refraction and Planar Focusing Based on Parity-Time Symmetric Metasurfaces", Physical Review Letters (2014), vol. 113, pp. 023903 - DOI: 10.1103/PhysRevLett.113.023903.

[17] R. Fleury, D. L. Sounas, and A. Alù, "An Invisible Acoustic Sensor Based on 
Parity-Time Symmetry”, Nature Communications (2015), vol. 6, pp. 5905 - DOI: 10.1038/ncomms6905.

[18] A. Ruschhaupt, F. Delgado, and J. G.Muga, "Physical realization of PT -symmetric potential scattering in a planar slab waveguide”, Journal of Physics A (2005), vol. 38, pp. L171-L176 - DOI: 10.1088/0305-4470/38/9/L03.

[19] R. El-Ganainy, K.G. Makris, D.N. Christodoulides, and Z.H. Musslimani, "Theory of coupled optical PT -symmetric structures", Optics Letters (2007), vol. 32, 2632-2634 -DOI: 10.1364/OL.32.002632.

[20] K.G.Makris, R. El-Ganainy, D.N. Christodoulides, and Z.H. Musslimani, "Beam dynamics in PT symmetric optical lattices", Physical Review Letters (2008), vol.100, pp.103904 - DOI: 10.1103/PhysRevLett.100.103904.

[21] S. Longhi, "Bloch oscillations in complex crystals with PT symmetry", Physical Review Letters (2009), vol. 103, pp. 123601 - DOI: 10.1103/PhysRevLett.103. 123601.

[22] M.V. Berry, "Physics of non-Hermitian degeneracies", Czech. Journal of Physics (2004), vol. 54, 1039-1047 - DOI: 10.1023/B:CJOP.0000044002.05657.04.

[23] W.D. Heiss, "The physics of exceptional points", Journal of Physiscs A (2012), vol. 45, pp. 444016 - DOI: 10.1088/1751-8113/45/44/444016.

[24] S. Longhi, "Spectral singularities and Bragg scattering in complex crystals", Physical Review A (2010), vol. 81 pp. 022102 - DOI: 10.1103/PhysRevA.81. 022102.

[25] S. Longhi, "Optical Realization of Non-Hermitian Quantum Mechanics", Physical Review Letters (2010), vol. 105, pp. 013903 - DOI: 10.1103/PhysRevLett.105. 013903.

[26] S. Longhi, "Coherent perfect absorption in a homogeneously broadened two-level medium”, Physical Review A (2011), pp. 055804 - DOI: 10.1103/PhysRevA.83. 055804.

[27] S. Longhi, L. Feng, "PT-symmetric microring laser- absorber", Optics Letters (2014), vol. 39 pp. 5026-5029 - DOI: 10.1364/OL.39.005026.

[28] Z.J. Wong, Y.-L. Xu, J. Kim, K. O’Brien, Y. Wang, L. Feng, and X. Zhang, "Lasing and anti-lasing in a single cavity", Nature Photonics (2016), vol. 10, pp. 796-801 - DOI: 10.1038/nphoton.2016.216.

[29] Z. Lin, H. Ramezani, T. Eichelkraut, T. Kottos, H. Cao, and D.N. Christodoulides, "Unidirectional Invisibility Induced by PT-Symmetric Periodic Structures", Physical Review Letters (2011), vol. 106, pp. 213901 - DOI: 10.1103/PhysRevLett.106.213901.

[30] S. Longhi, "Invisibility in PT -symmetric complex crystals", Journal of Physics A (2011), vol. 44, pp. 485302 - DOI: 10.1088/1751-8113/44/48/485302.

[31] S. Longhi, "A unidirectionally invisible PT -symmetric complex crystal with arbitrary thickness", Journal of Physics A (2014), vol. 47, pp. 485302 - DOI: 10.1088/1751-8113/47/48/485302.

[32] I. Fausto, "Fisica dell'invisibilità. Metamateriali e tecnologie del futuro" (Arance Editrice, Milano, 2013).

[33] S. Longhi, "Invisibility in non-Hermitian tight-biniding lattices", Physical Review A (2010), vol. 82, pp. 032111 - DOI: 10.1103/PhysRevA.82.032111. 
[34] S. Longhi, "Half-spectral unidirectional invisibility in non-Hermitian periodic optical structures”, Optics Letters (2015), vol. 40, pp. 5694-5697 - DOI: 10.1364/OL.40.005694.

[35] P. Miao, Z. Zhang, J. Sun, W. Walasik, S. Longhi, N.M. Litchinitser, and L. Feng, "Orbital angular momentum microlaser", Science (2016), vol. 353, pp. 464-467 DOI: 10.1126/science.aaf8533.

[36] S. Longhi and G. Della Valle, "Invisible defects in complex crystals", Annals of Physics (2013), vol. 334, pp. 35-46 - DOI: 10.1016/j.aop.2013.03.011.

[37] S.A.R. Horsley, M. Artoni, G.C. La Rocca, "Spatial Kramers-Kronig relations and the reflection of waves", Nature Photonics (2015), vol. 9, pp. 436-439 - DOI: 10.1038/nphoton.2015.106.

[38] S. Longhi, "Wave reflection in dielectric media obeying spatial Kramers-Kronig relations", EuroPhysics Letters (2015), vol. 112, pp. 64001 - DOI: 10.1209/02955075/112/64001.

[39] S.A.R. Horsley and S. Longhi, "Spatiotemporal deformations of reflectionless potentials”, Physical Review A (2017), vol. 96, pp. 023841 - DOI: 10.1103/ PhysRevA.96.023841.

[40] S. Longhi, "Bidirectional invisibility in Kramers-Kronig optical media", Optics Letters (2016), vol. 41, pp. 3727-3730 - DOI: 10.1364/OL.41.003727.

[41] S. Longhi, "Time-Reversed Optical Parametric Oscillation", Physical Review Letters (2011), vol. 107, pp. 033901 - DOI: 10.1103/PhysRevLett.107.033901.

[42] M.-A. Miri, M. Heinrich, and D.N. Christodoulides, "SUSY-inspired one-dimensional transformation optics”, Optica (2014), vol. 1, pp. 89-95 - DOI: 10.1364/OPTICA.1.000089.

[43] S. Longhi, "Supersymmetric transparent optical intersections", Optics Letters (2015), vol. 40, pp. 463-466 - DOI: 10.1364/OL.40.000463.

[44] S. Longhi, "Supersymmetric Bragg gratings", Journal of Optics (2015), vol.17, pp. 045803 - DOI: 10.1088/2040-8978/17/4/045803. 
